\title{
La réhabilitation prothétique d'une perte de substance vélo-palatine : à propos d'un cas clinique
}

\section{The prosthetic rehabilitation of a hard and soft palate defect: case report}

\author{
Résumé
}

\section{MOTS-CLEFS :}

- Prothèse vélo-palatine, édenté complet, carcinome épidermoïde

\section{KEYWORDS:}

- Soft and hard palate prosthesis, full toothless, squamous cell carcinoma

$\operatorname{AOS} n^{\circ} 286-2017$ total irradié.
La prothèse vélo-palatine est l'une des solutions thérapeutiques de plus en plus utilisée pour les cas de pertes de substance vélaires et palatines post-chirurgicales. La réalisation de ce type de réhabilitation nécessite une grande habilité de la part du praticien et doit être effectuée dans le cadre d'une équipe pluridisciplinaire incluant le chirurgien, l'orthophoniste, le psychologue et le spécialiste en prothèse maxillo-faciale.

À travers un cas clinique pris en charge au Centre de Consultation et des Traitements Dentaires de Rabat, les auteurs se permettent de faire le point sur les différentes étapes cliniques et de laboratoire de réalisation d'une prothèse vélo-palatine chez un patient édenté

Les défauts vélaires et palatins altèrent véritablement les fonctions oro-pharyngées, en l'occurrence la respiration, la déglutition et surtout la phonation ; le spécialiste en prothèse maxillo-faciale doit mettre toute sa vigilance pour rétablir au maximum ces fonctions.

Le patient pris en charge comporte plusieurs difficultés pour une telle réhabilitation. En plus de la perte de substance qui affecte le voile et le palais dur, le patient présente une édentation complète, des complications liée ò la radiothérapie notamment la xérostomie et la forte sensibilité des structures para-prothétiques ainsi qu'une limitation excessive de l'ouverture buccale suite à la cicatrisation post-chirurgicale.

Ce terrain, certes difficile, est traité selon un concept global, en prenant en considération tous ces obstacles avec des techniques très spécifiques, permettant d'atteindre les résultats désirés sur le plan esthétique et fonctionnel.

\section{Abstract}

The soft and palate prosthesis is one therapeutic solutions increasingly used for any loss of velar and palatal postsurgical substances. The realization of such rehabilitation requires great skill on the part of the practitioner and should be performed as part of a multidisciplinary team including surgeons, speech therapist, psychologist and specialist in maxillofacial prosthesis.

Through a clinical case supported at the Consultation Center and Dental Treatments of Rabat, the authors make it possible to review the different clinical stages and laboratories for making a soft and hard palate prosthesis in a toothless total irradiated patient.

Velar and palatal defects truly alter oropharyngeal functions, namely breathing, swallowing and phonation especially; the specialist in maxillofacial prosthesis should put all its vigilance to restore up these functions.

The patient has supported several difficulties for such a rehabilitation. In addition to the defect that affects the soft and the hard palate, the patient presents a complete tooth loss, complications of post radiotherapy including xerostomia and the high sensitivity of para prosthetic structures and excessive limitation of mouth opening after to the post-surgical healing.

This field certainly difficult, is treated as an overall concept, taking into consideration all these obstacles with very specific techniques to achieve the desired results on the aesthetic and functional terms. 


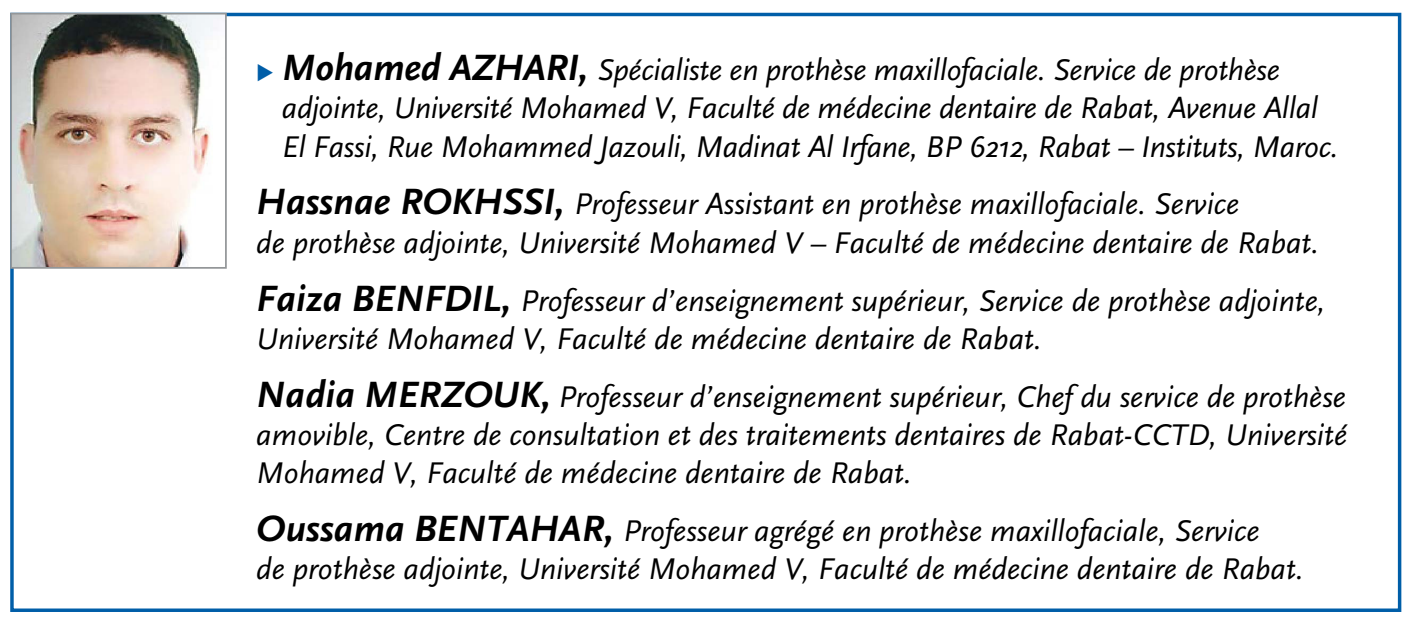

\section{INTRODUCTION}

La prothèse vélo-palatine est définie comme étant une prothèse remplaçant à la fois le palais dur et le palais mou ou voile du palais ; cette prothèse reste une solution thérapeutique à envisager après une chirurgie de résection siégeant au niveau vélaire.

Le palais dur forme le toit de la cavité buccale qui la sépare des fosses nasales et se continue postérieurement par le voile du palais qui se présente sous forme d'un rideau mobile permettant de régulariser l'automatisme du système phonatoire; la perte de cette structure, va créer un véritable problème fonctionnel par perturbation de la physiologie de la déglutition, la respiration et surtout la phonation.

Les pertes de substance vélo-palatines après exérèse tumorale, peuvent avoir plusieurs formes selon leurs sièges et leurs étendues. Tant que le support osseux et dentaire est préservé lors de la chirurgie, la reconstitution par prothèse reste de pronostic favorable. Quand ce défaut palatin est associé à une édentation complète et une radiothérapie, la restauration prothétique devient de plus en plus difficile et nécessite l'usage des techniques plus élaborées pour sa réalisation.

À travers cette mise au point, on va essayer de décrire les différentes étapes cliniques et de laboratoire d'élaboration d'une prothèse vélo-palatine, ainsi que la spécificité de la prise en charge prothétique d'un édenté total irradié présentant une large perte de substance vélo-palatine.

\section{OBSERVATION CLINIQUE}

\section{Examen clinique}

Il s'agit de Mr M.S. âgé de 70 ans, adressé par le service de chirurgie maxillo-faciale au service de Prothèse Adjointe au Centre de Consultation et des Traitements Dentaires de Rabat, après résection d'un carcinome épidermoïde siégeant au niveau maxillaire droit pour une réhabilitation prothétique esthétique et fonctionnelle. L'examen clinique permet de déceler un affaissement de l'hémi-face droite, une ligne bicommissurale qui converge à gauche par rapport à la ligne bipupillaire, une limitation de l'ouverture buccale et une perte de substance intéressant la totalité du maxillaire droit, l'os palatin droit et la moitié droite du voile du palais ( $\mathrm{Cl}$ IIb de Kolb [1]) l'hémi-os palatin droit et la moitié du voile du palais (moitié droite du palais dur et du palais mou). Ceci a entrainé des troubles esthétiques et fonctionnels trop importants (Fig. 1-2). À noter que le temps écoulé après exérèse tumorale était de plus d'une année et que le patient a reçu une dose totale de 60 Gy par téléradiothérapie.

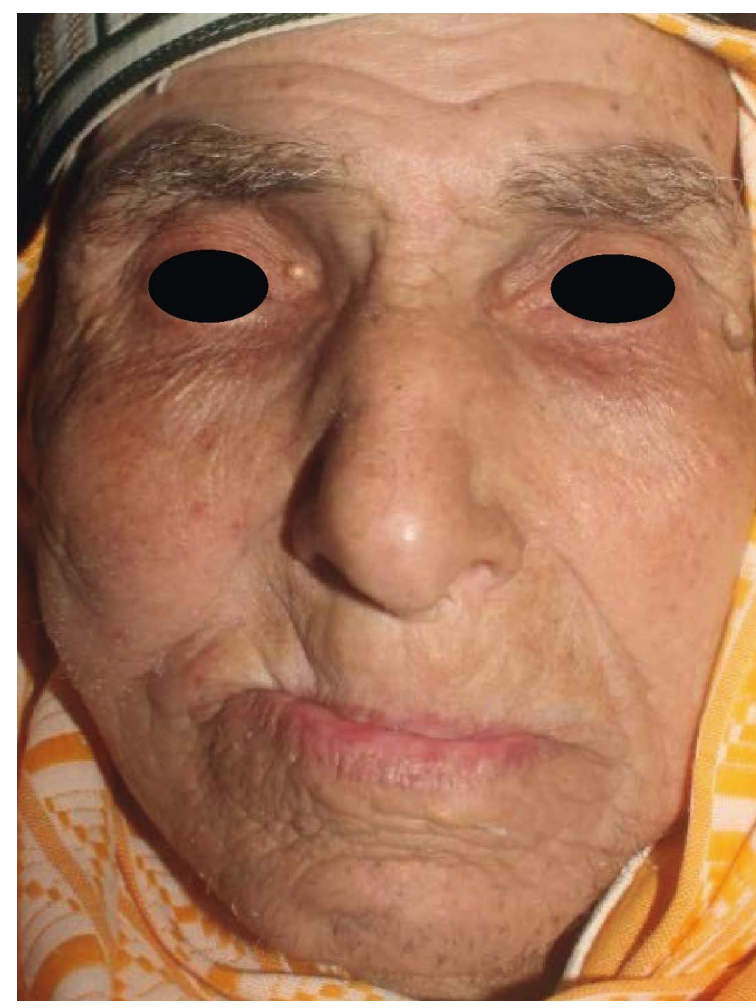

Fig. 1 : Examen clinique exo-buccal. 


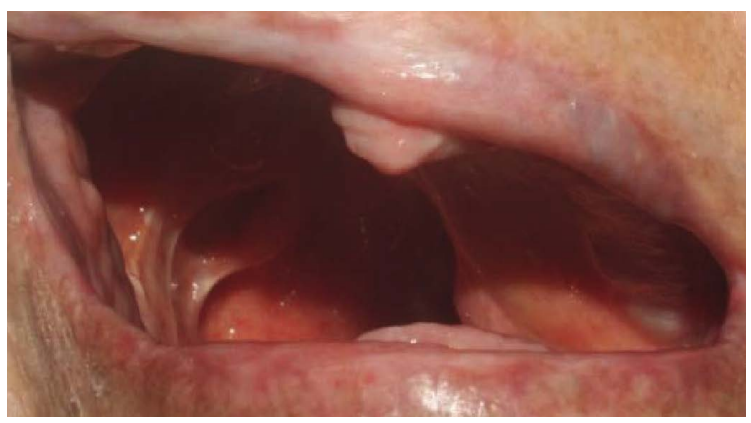

$\triangle$ Fig. 2 : Examen clinique endo-buccal : perte de substance vélopalatine II b de Kolb [1].

\section{Étapes prothétiques}

Étant donné que le patient est reçu un an après la fin de la radiothérapie et que le palais est stable de point de vue cicatrisation, la décision de réalisation d'une prothèse d'usage a été prise. La réalisation prothétique va être abordée selon trois segments prothétiques successifs et liés entre eux : la prothèse dentaire qui est dans ce cas une prothèse amovible complète (PAC), une prothèse obturatrice d'usage vu le temps écoulé après la chirurgie ( 1 an) et un obturateur vélo-pharyngien (OVP); l'ensemble de ces segments constitue la prothèse vélo-palatine (PVP).

\section{Réalisation de la prothèse amovible complète associée à un obturateur palatin d'usage $(P A C+O P U)$}

Les empreintes maxillaires sont réalisées en trois temps :

I Premier temps : il s'agit d'une empreinte préliminaire, réalisée à l'aide d'alginate avec un porteempreinte de série modifié en regard de la perte de substance à l'aide de la pâte thermoplastique de Kerr ${ }^{\circledast}$ (Fig. 3). Cette empreinte (Fig. 4) va permettre d'enregistrer la surface d'appui maxillaire et palatine alors que la portion vélaire reste difficile à enregistrer à ce stade (Fig. 5), d'où l'importance de cette empreinte pour pouvoir confectionner un porte-empreinte individuel (PEI) où on effectue une extension vers le moignon vélaire, ce PEI est dit PEI primaire (Fig. 6). $\checkmark$ Deuxième temps : c'est une empreinte primaire statique à l'alginate, réalisée avec le porte-empreinte individuel primaire préalablement confectionné (Fig. 7). Cette empreinte a pour but d'affiner au maximum l'enregistrement de la surface d'appui (Fig. 8) et de permettre la réalisation d'un PEI secondaire (Fig. 9) qu'on va utiliser pour l'empreinte anatomo fonctionnelle. I Troisième temps : c'est l'étape de l'empreinte anatomofonctionnelle qui est composée d'une empreinte

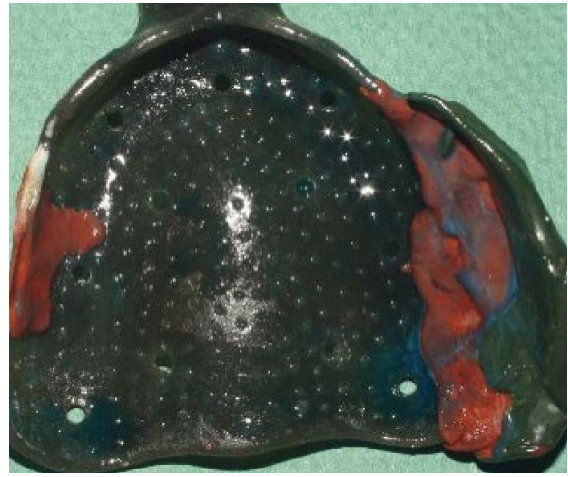

$\triangle$ Fig. 3 : Porte-empreinte de série modifié en rapport avec la perte de substance.

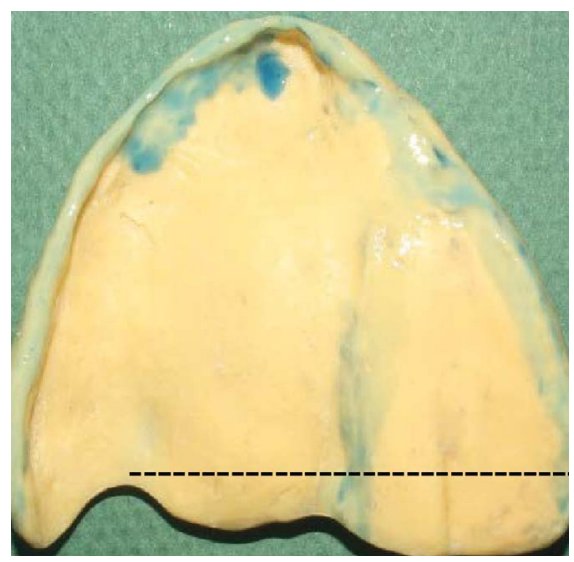

$\triangle$ Fig. 6 : Porte-empreinte individuel primaire.

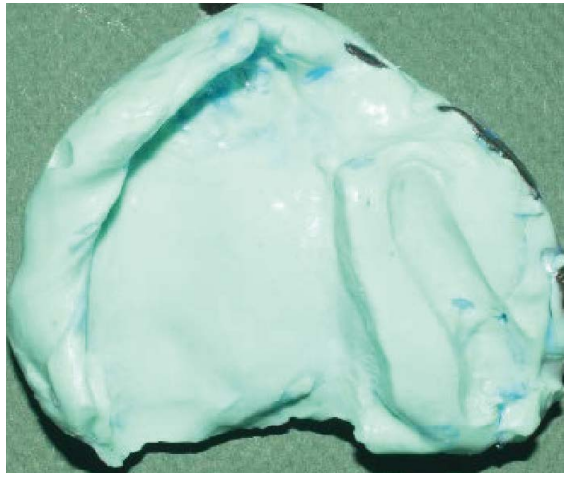

Fig. 4 : Empreinte primaire maxillaire réalisée à l'aide de l'alginate.

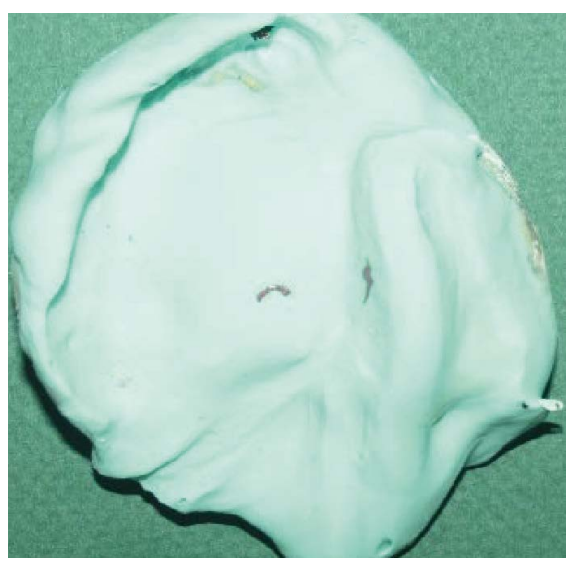

$\triangle$ Fig. 7 : Empreinte primaire à l'alginate enregistrant de manière plus précise la région vélaire.

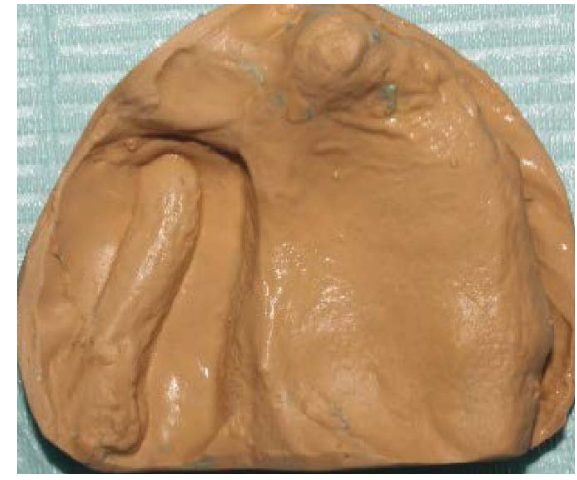

$\triangle$ Fig. 5 : Modèle primaire maxillaire avec région vélaire manquante.

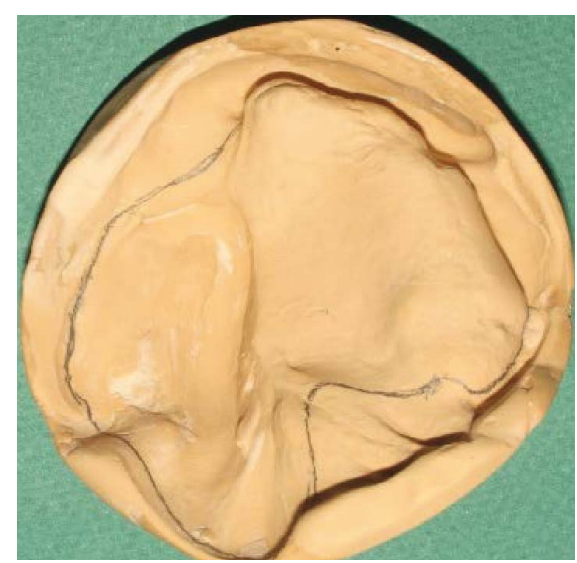

$\triangle$ Fig. 8 : Modèle primaire avec région vélaire bien enregistrée. 
du joint périphérique avec un polyéther (Fig. 10) et d'une empreinte globale avec un polysulfure (Fig. 11). Les empreintes mandibulaires sont réalisées en deux temps, l'empreinte primaire muco-statique à l'alginate permettant d'obtenir un modèle primaire (Fig. 12) et l'empreinte secondaire anatomo-fonctionnelle obéissant aux mêmes principes des empreintes en prothèse amovible complète, la seule spécificité étant l'usage des matériaux spécifiques pour des terrains irradiés en l'occurrence les polyéthers et les polysulfures en évitant donc d'utiliser les matériaux comme la pâte d'oxyde de zinc- eugénol contre-indiquée chez ce type de patients présentant une sécheresse buccale et une fragilité de la muqueuse buccale (Fig. 13-14-15). La détermination du plan d'occlusion en orientation et situation suit les critères référentiels esthétique et fonctionnel de PAC (Fig. 16-17), la largeur et la hauteur du bourrelet en regard de la perte de substance

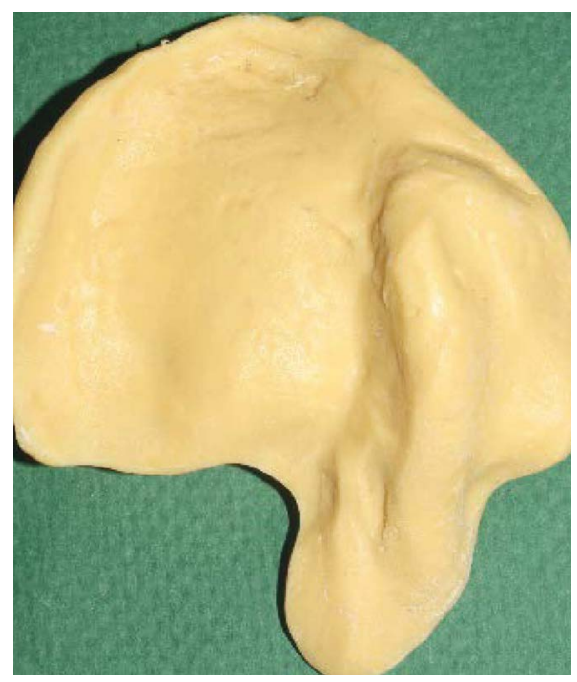

$\triangle$ Fig. 9: Porte-empreinte individuel secondaire utilisé pour la réalisation de l'empreinte anatmofonctionnelle.

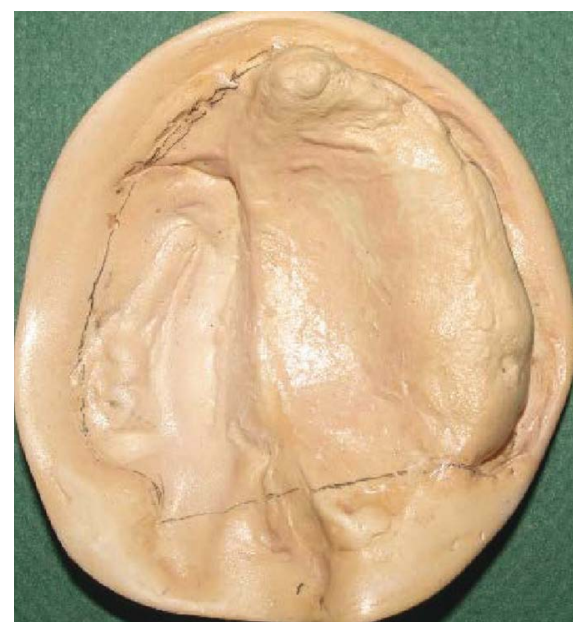

$\triangle$ Fig. 12 : Modèle de travail maxillaire.

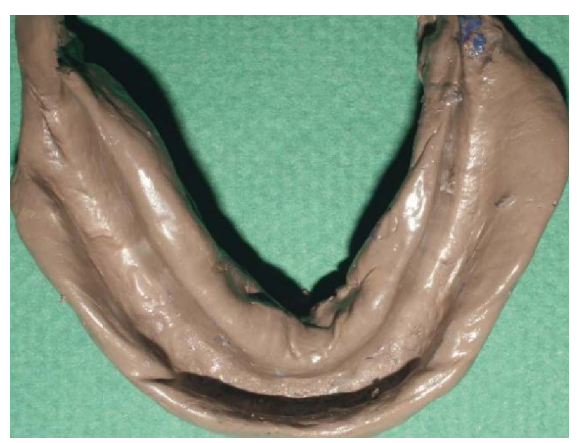

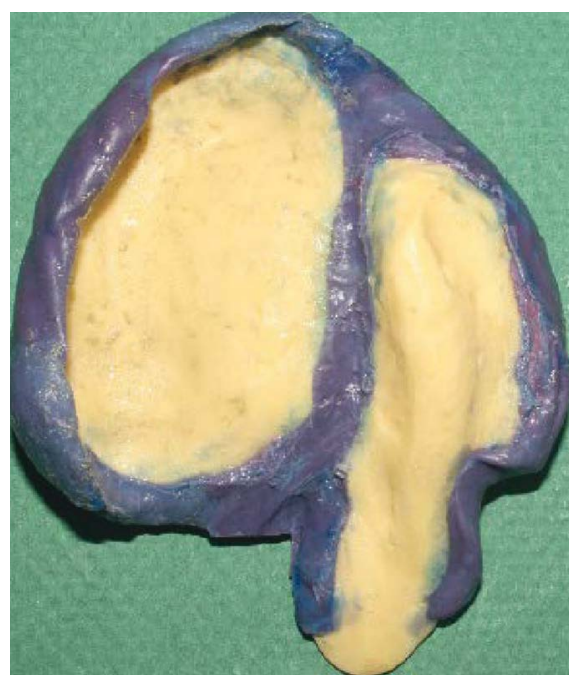

$\triangle$ Fig. 10 : Empreinte du joint périphérique à l'aide d'un polyéther.

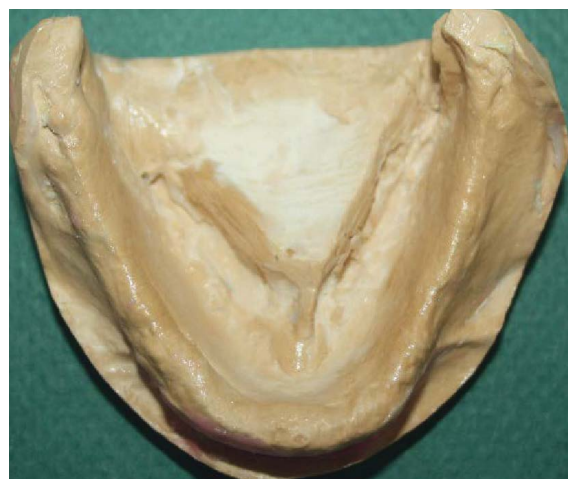

$\triangle$ Fig. 13 : Modèle primaire mandibulaire.

$\succ$ Fig. 15 : Empreinte anatomo-fonctionnelle de la surface d'appui mandibulaire.

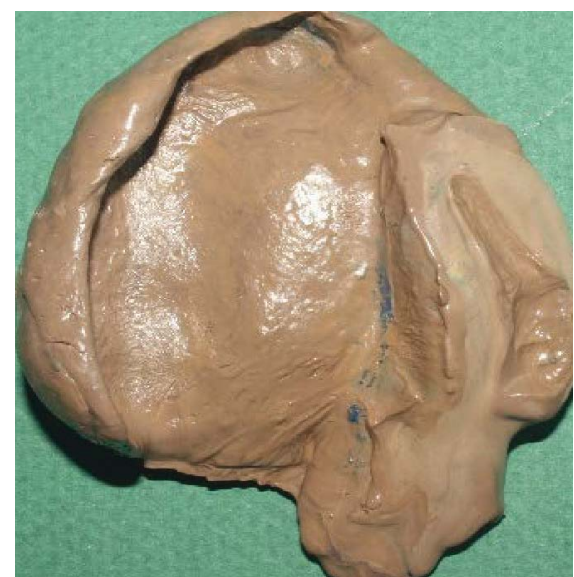

$\triangle$ Fig. 11 : Empreinte globale de la surface d'appui palatine à l'aide d'un polysulfure de basse viscosité.

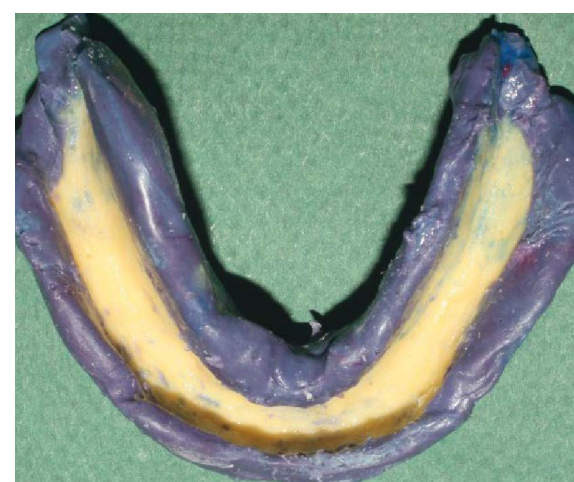

$\triangle$ Fig. 14 : Empreinte du joint périphérique à l'aide d'un polyéther.

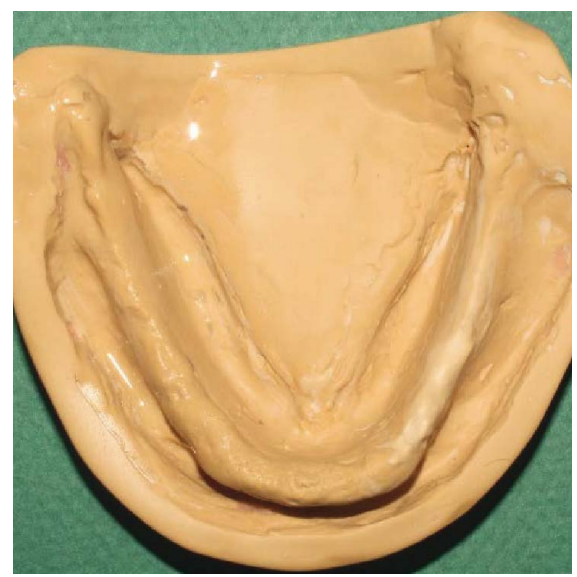



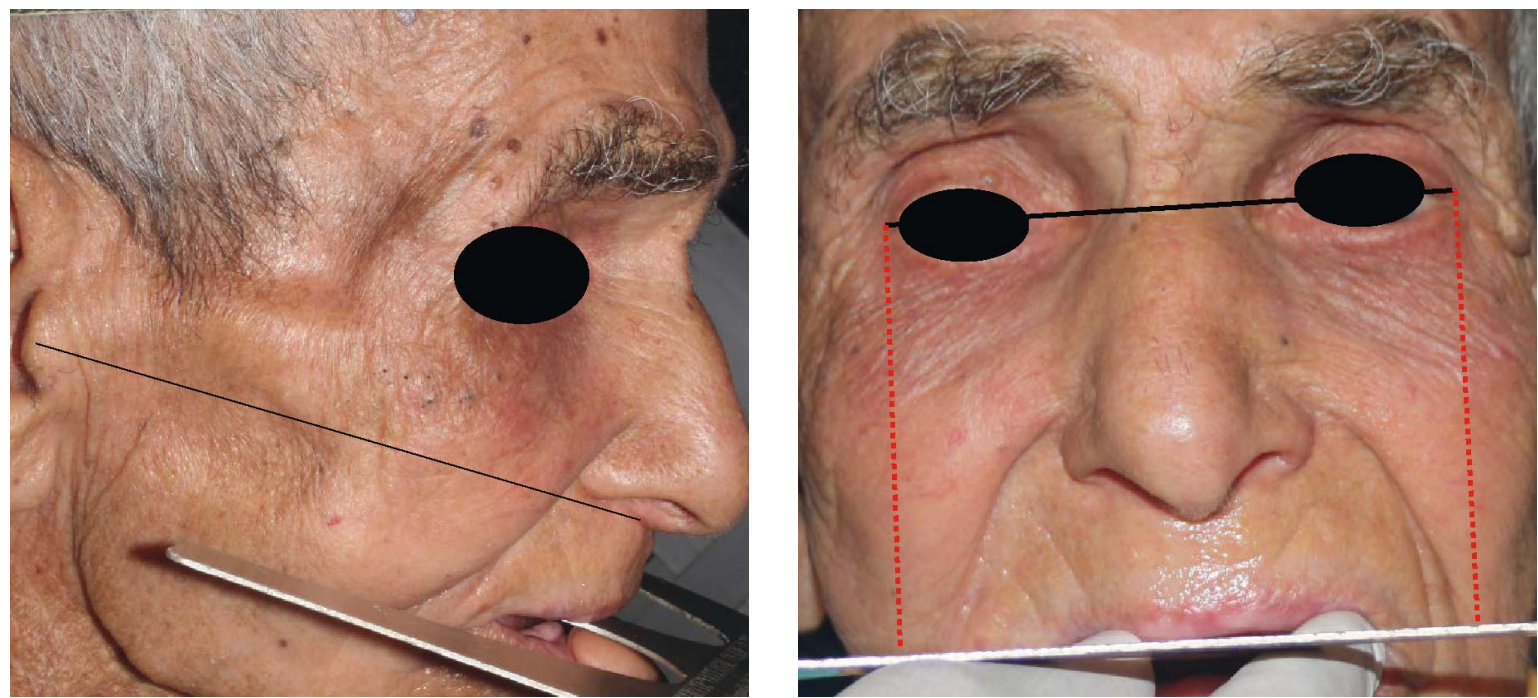

$\triangle$ Fig. 17-18 : Détermination de l'orientation du plan d'occlusion par rapport au plan bi-pupillaire au niveau antérieur et au plan de Camper au niveau postérieur.

sont suffisamment importante pour pouvoir assurer d'une part le soutien de la musculature périphérique et d'autre part le remplacement de l'os maxillaire, l'os alvéolaire et l'arcade dentaire absents (Fig. 18). La référence articulaire utilisée est la relation centrée. La dimension verticale est déterminée en fonction de l'harmonie du visage, elle doit étre correctement évaluée voire sous-évaluée mais jamais surévaluée pour éviter les lésions de la surface d'appui. La limitation de l'ouverture buccale est gérée dans notre situation par section de la maquette d'occlusion mandibulaire à l'aplomb de l'éminence piriforme mandibulaire pour pouvoir effectuer l'enregistrement du rapport maxillo mandibulaire dans de bonnes conditions (Fig. 19-20).

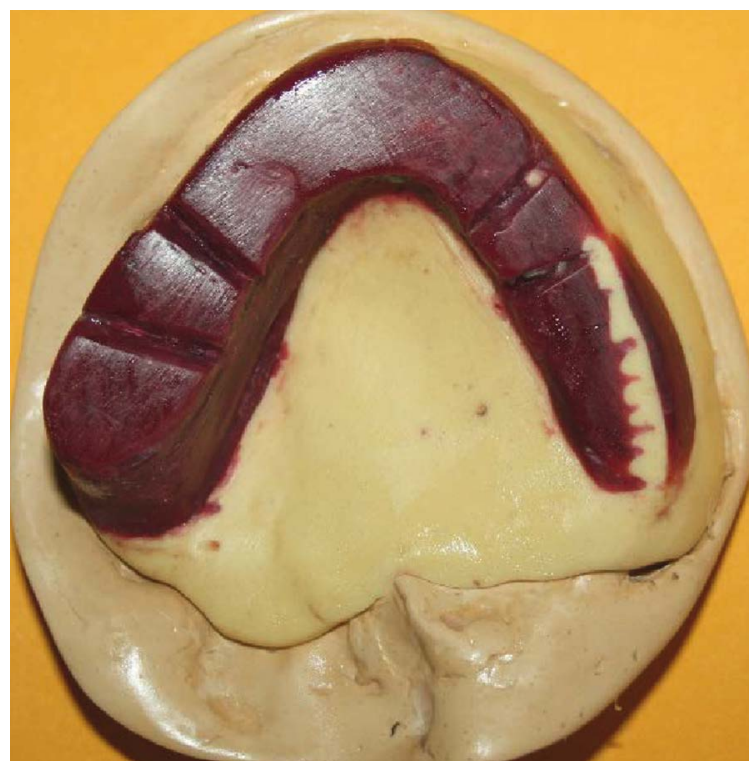

Fig. 19-20 : Enregistrement du rapport inter maxillaire.
Le montage de dents prothétiques doit respecter le concept occlusal cinématique spécifique à la prothèse amovible complète notamment l'occlusion intégralement équilibrée (Fig. 21-22-23).

L'étape d'insertion en bouche doit commencer par un contrôle des zones de compression et de surextension à l'aide d'un élastomère de basse viscosité. Puis un rebasage de l'intrados prothétique (PAC + OPU) avec une résine à prise retardée (FITT de Kerrø) qui a pour but de protéger la surface d'appui contre tout traumatisme du à l'inégalité de sustentation par présence d'un défaut palatin important (hémi-palais) et aux charges occlusales obliques déstabilisatrices à ce niveau, mais aussi de rendre confortable le port de la prothèse du moins durant les premières séances en

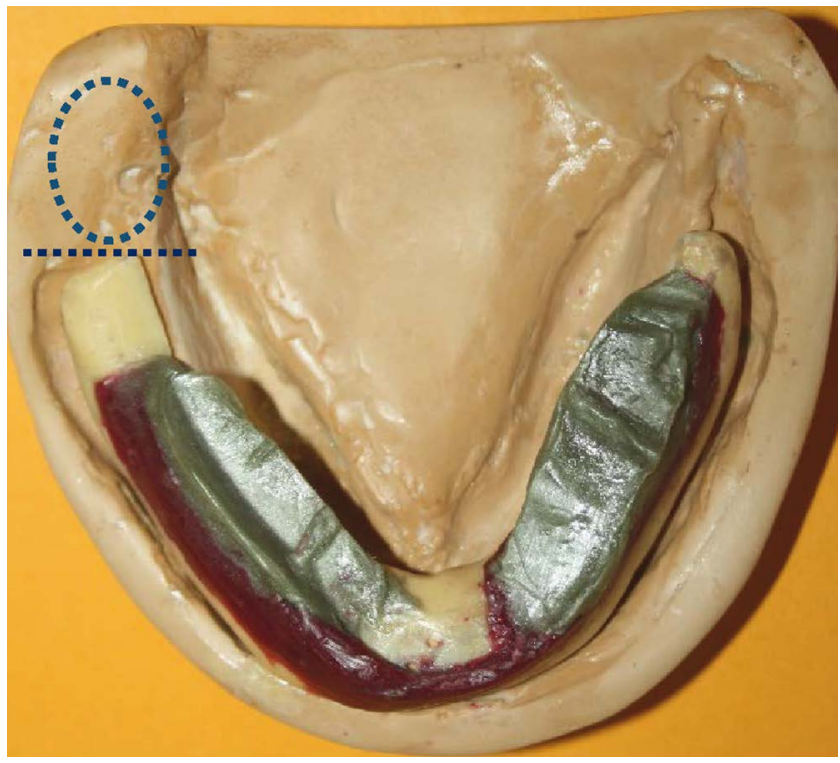



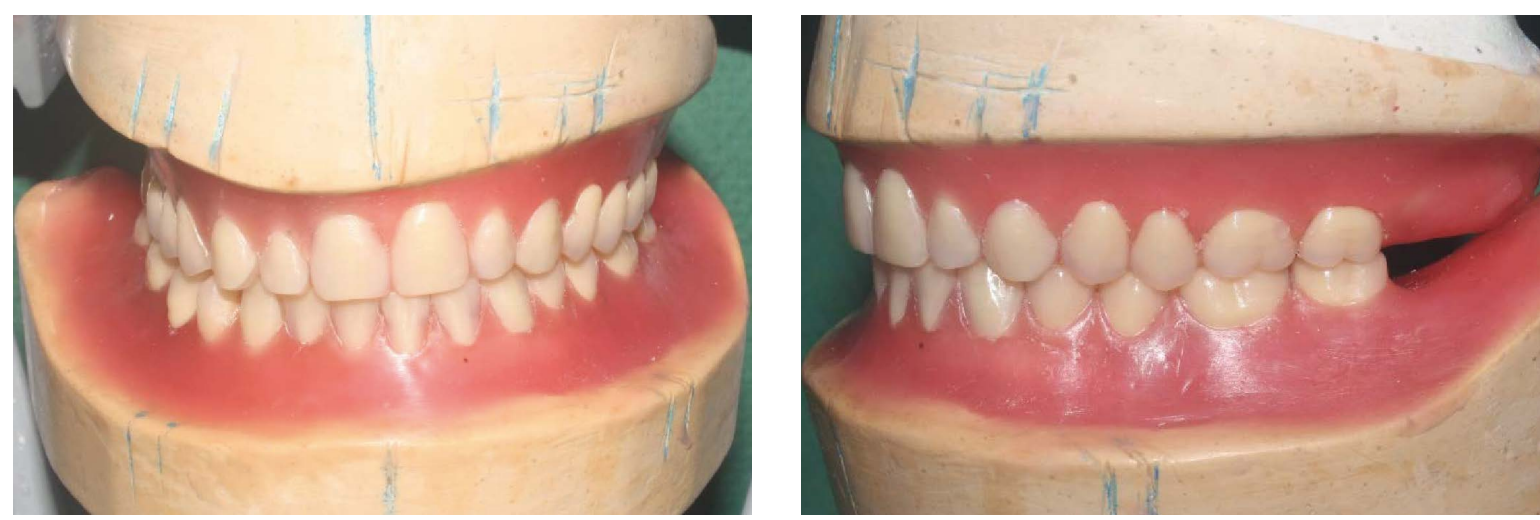

$\triangle$ Fig. 21-22 : Montage des dents prothétiques.
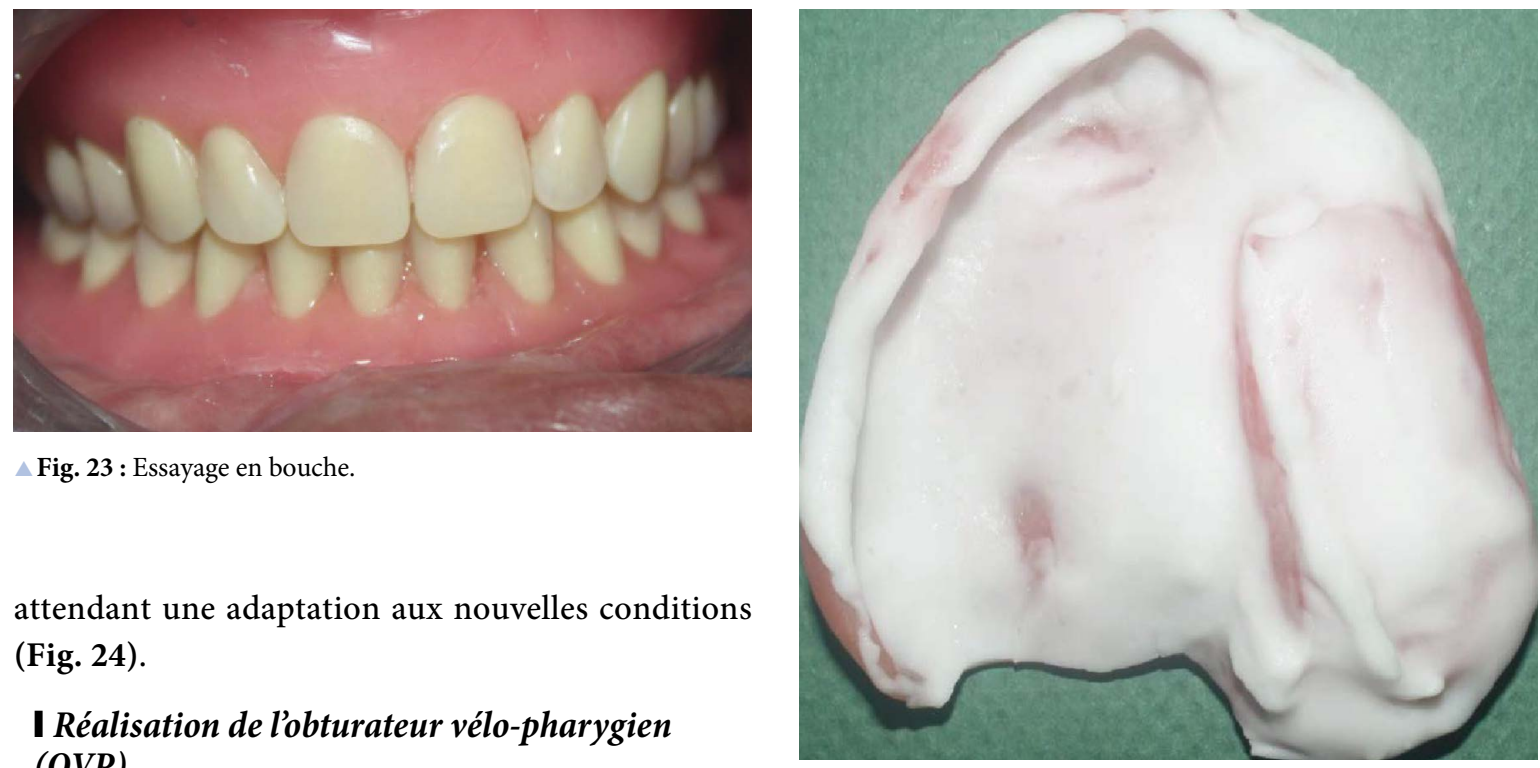

attendant une adaptation aux nouvelles conditions (Fig. 24).

\section{Réalisation de l'obturateur vélo-pharygien (OVP)}

Au niveau du bord postérieur de la première prothèse (PAC + OPU), on solidarise trois fils métalliques avec une résine chémopolymérisable (Fig. 25), puis on vérifie la prothèse en bouche et tout contact des fils avec les parois pharyngiennes est à rectifier avec une pince universelle en veillant à laisser un espace d'au moins $5 \mathrm{~mm}$ au repos et en fonction. Ces fils sont flexibles bouclés dans leurs extrémités pour ne pas traumatiser les parois pharyngiennes, puis enduits par le matériau de modelage qui est dans notre situation clinique la pâte thermoplastique de $\operatorname{Kerr}^{\circledR}$ (Fig. 26). Ce prolongement doit respecter au maximum les bords du moignon vélaire et la paroi postérieure du pharynx notamment un repli transversal soulevé par les follicules lymphoïdes et reliant les deux orifices tubaires qu'on appelle le bourrelet de Passavant. Il faut absolument souligner que la manipulation de la pâte thermoplastique doit être faite avec beaucoup de précaution chez un patient irradié en évitant plus particulièrement de la surchauffer.

Fig. 25 : Solidarisation de trois fils métalliques flexibles au niveau du bord postérieur de la prothèse obturatrice maxillaire.

$\triangle$ Fig. 24 : Pose de la prothèse obturatrice avec intrados enduit du matériau de mise en condition tissulaire.

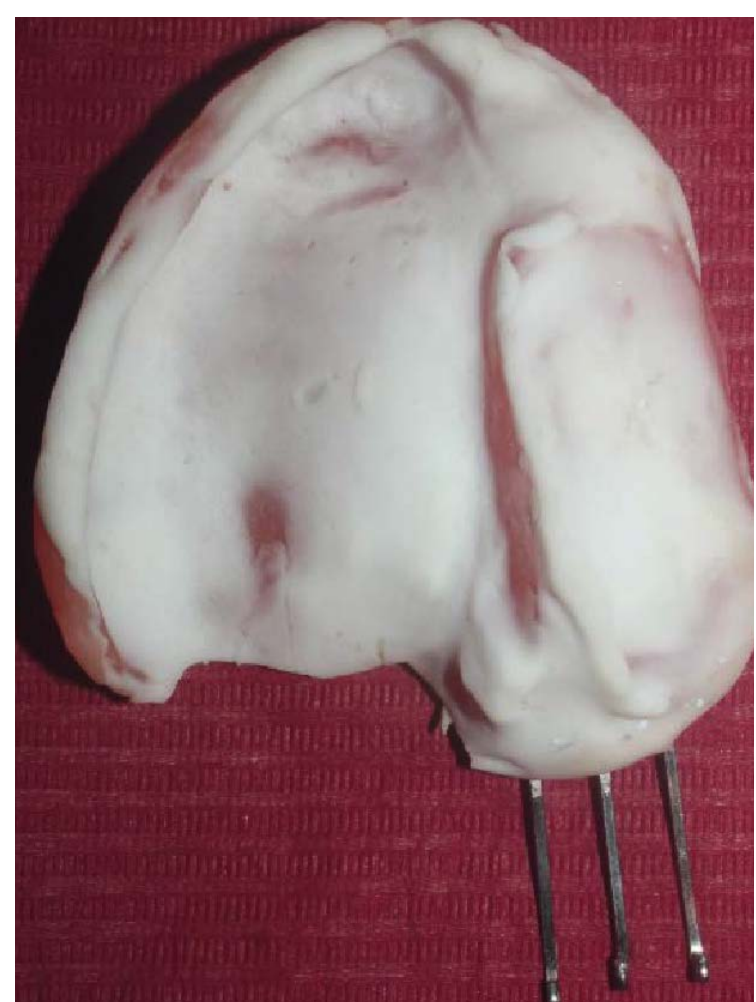




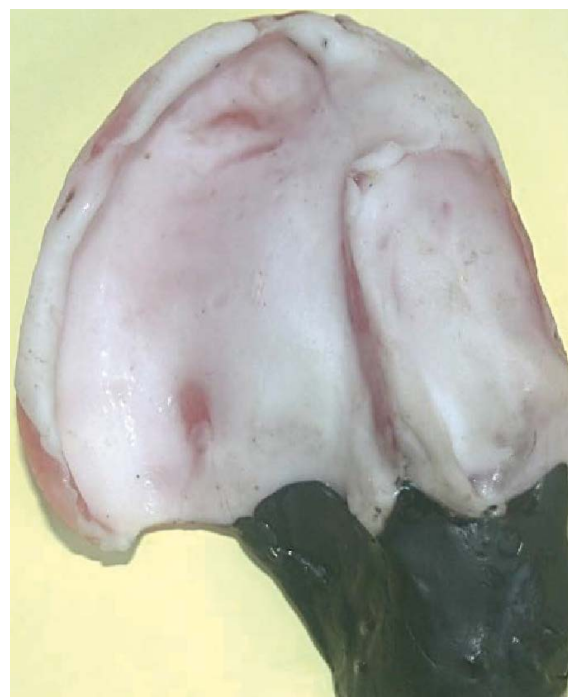

$\triangle$ Fig. 26: Une « raquette» en pate de Kerr ${ }^{\circledR}$ est réalisée en l'adaptant au maximum au moignon vélaire.

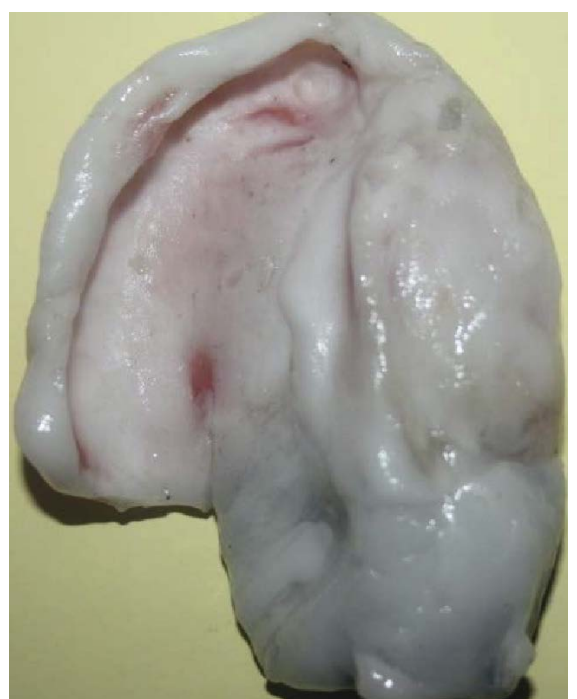

$\triangle$ Fig. 27 : Intrados de la prothèse obturatrice et le prolongement vélaire sont enduits par une résine à prise retardée (FITT ${ }^{\circledR}$ de Kerr) : empreinte fonctionnelle vélaire.

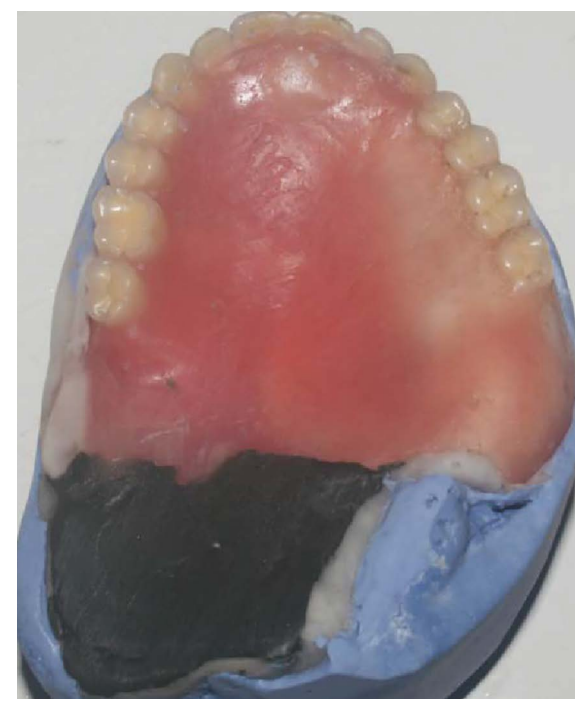

$\triangle$ Fig. 28 : Rebasage au laboratoire.
L'intrados de la prothèse obturatrice et son prolongement vélaire sont garnis avec une résine à prise retardée (Fig. 27), puis la prothèse est insérée en bouche et on demande au patient d'effectuer les mouvements fonctionnels suivants : mobiliser la tête de manière circulaire de gauche à droite, pencher la tête au maximum vers l'avant et vers l'arrière, prononcer «ah » grave et avaler. La prothèse est envoyée par la suite pour un rebasage au laboratoire (Fig. 28).

Après la pose finale de la prothèse vélo-palatine (Fig. 29-30-31), des séances de contrôle clinique et radiologique sont programmées pour s'assurer de la bonne adaptation de l'OVP et son intégration physiologique par rapport au moignon vélaire et aux parois pharyngiennes en demandant au patient d'effectuer les mouvements précédemment décrits.

\section{DISCUSSION}

Dans la littérature, il n'y a pas de classification limitée aux pertes de substances vélo-palatines. Mais on peut utiliser la classification proposée par Kolb F (Fig. 32) [1], inspirée des travaux d'Urken et qui est différente des autres classifications par sa description lésionnelle non limitée au maxillaire. Elle prend en compte la totalité des structures osseuses, cartilagineuses, cutanées, musculaires, muqueuses et nerveuses de l'étage moyen, ainsi que les fonctions principales. Cette classification n'a pas pour objectif d'établir un algorithme décisionnel, elle rend possible l'analyse de la perte de substance en termes pratiques de reconstruction. L'identification des tissus et fonctions atteints permet de déterminer les objectifs et hiérarchiser les priorités

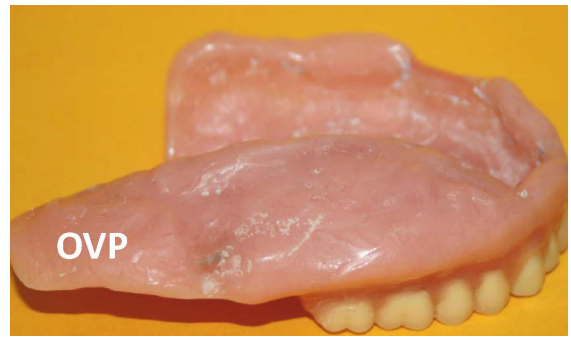

$\triangle$ Fig. 29-30-31 : Prothèse vélo-palatine (PVP) avec ses trois segments :

Prothèse amovible complète (PAC); Prothèse obturatrice d'usage (OPU) ; Obturateur vélo-pharyngien (OVP).
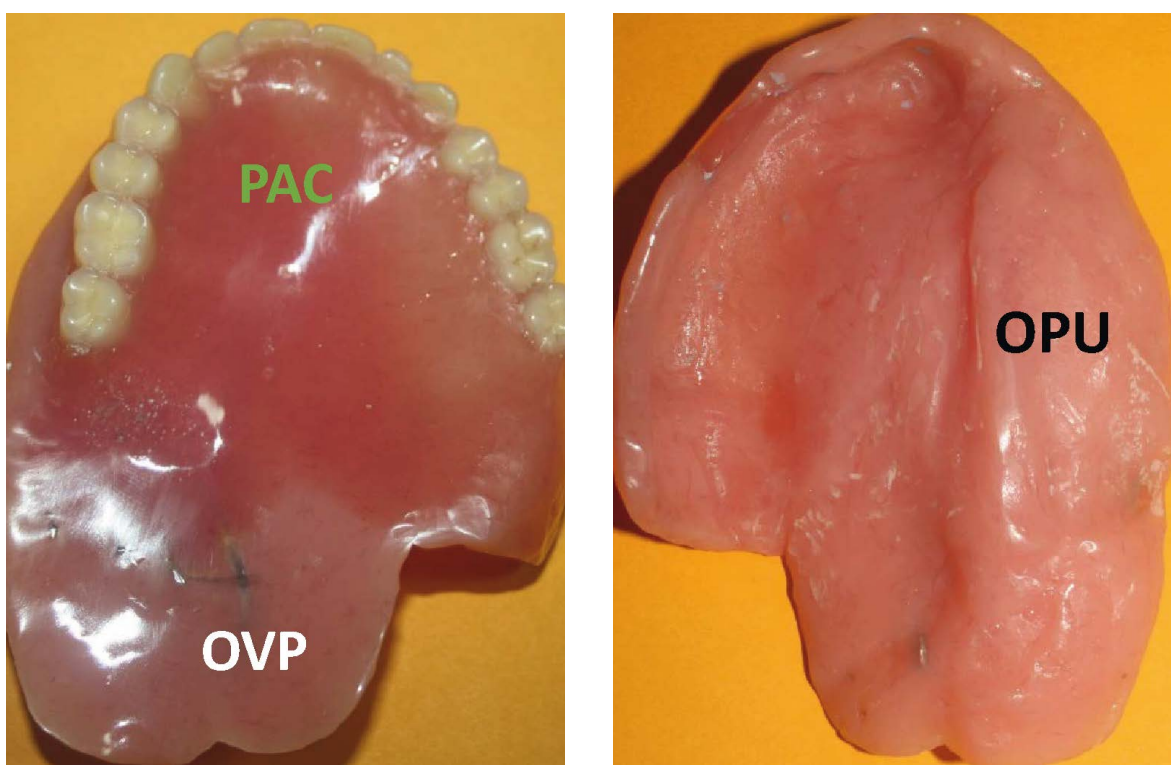


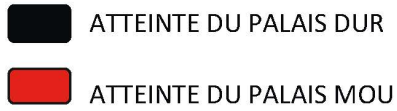

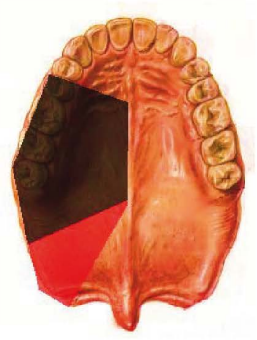

CLASSE I

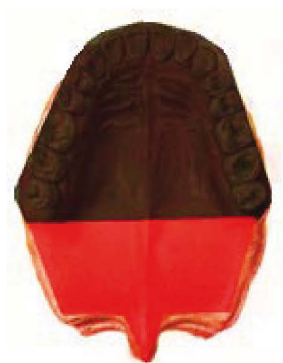

CLASSE IV

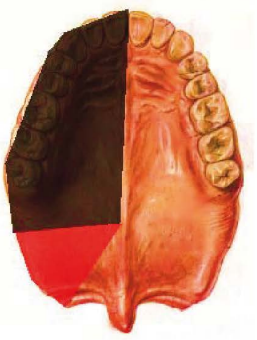

CLASSE II

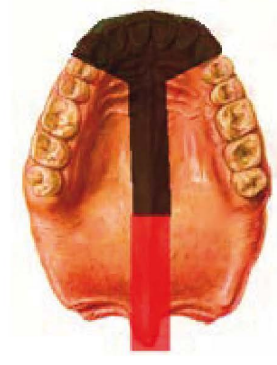

CLASSE V

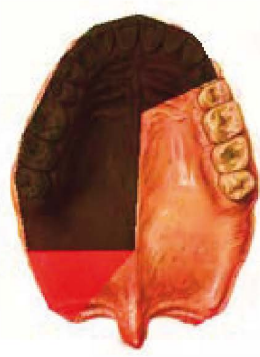

CLASSE III

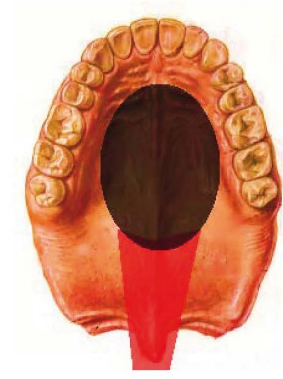

CLASSE VI $\triangle$ Fig. 32 : Classification des pertes de substance vélo-palatines selon KOLB.

de la reconstruction, de prévoir les séquelles, et ainsi de proposer une prise en charge adaptée. Dans ce sens, c'est l'étendue de la résection, la nature de la dentition restante et la qualité du moignon palatin, qui permettent le choix de la technique de reconstruction soit par la réhabilitation prothétique ou par les lambeaux. Dans la classification globale de Kolb des pertes de substance de la région cervicofaciale on ne va s'intéresser qu'au volet en rapport avec la voûte palatine. Cette dernière est l'une des régions du massif facial la plus fréquemment atteinte lors de la chirurgie d'exérèse tumorale. Sa localisation à la jonction entre les cavités sinuso-nasales et oropharynx, et sa relative simplicité anatomique ainsi que le caractère standardisé de sa chirurgie carcinologique ont rendu possible la définition de six classes principales de résection palatine, désignées de I à VI. L'atteinte isolée du palais osseux correspond à une classe « $\mathrm{a}$ », et la classe « $\mathrm{b}$ » à une atteinte concomitante du voile du palais. À titre d'exemple, la classe II b est une perte de substance intéressant l'hémi-palais dur et l'hémi-voile du palais. \Classe I : perte de substance palatine unilatérale située en arrière de la canine.

$\checkmark$ Classe II : résection de l'hémi-voûte palatine.

\Classe III : résection subtotale du palais osseux, incluant la canine controlatérale.
Classe IV : résection totale de la voûte palatine.

I Classe V : perte de substance limitée au prémaxillaire.

IClasse VI : perte de substance centro-palatine.

Dans les situations des classes I, II (surtout IIa), V et VI, la reconstruction prothétique est favorable. Par contre dans les classes III et IV, la chirurgie est préférable à travers des lambeaux libres notamment le lambeau scapulodorsal.

La principale cause de défaut palatin est acquise ou congénitale. La résection tumorale, en particulier une tumeur maligne, est la cause la plus fréquente d'un défaut palatin acquis ; tandis que la fente palatine est la principale cause des défauts palatins congénitaux. Le défaut palatin peut être réparé par la chirurgie reconstructrice et/ou une prothèse maxillo-faciale. Chez les patients avec une tumeur, il est admis que la réhabilitation prothétique est généralement préférable à la chirurgie réparatrice, puisqu'elle facilite l'inspection du tissu résiduel après la chirurgie [2]. En effet une éventuelle récidive peut être précocement décelée chez ces patients en post-opératoire, chose qui n'est pas aisée cliniquement après la chirurgie reconstructrice des défauts vélo-pharyngés. Chaque fois que le patient souffre d'un défaut palatin, une fuite nasale de la nourriture et des liquides, et un hypernasonnement de la parole deviennent inévitables [3]. Par conséquent ceci va constituer un véritable handicap pour les patients dans leurs activités quotidiennes. Chez les patients ayant à la fois le défaut vélo-palatin et l'absence totale de dents supérieures, cette édentation totale maxillaire complique encore la confection de la prothèse vélo-palatine.

Le palais mou agit comme un séparateur dynamique entre la cavité buccale et nasale. Le voile, les parois latérales et postérieures du pharynx forment le sphincter vélo-pharyngé (SVP), c'est une sorte de valve musculaire en trois dimensions [4]. La fermeture adéquate de ce sphincter est nécessaire lors de la déglutition et de la phonation en particulier la prononciation des consonnes.

L'altération de la phonation suite à la résection vélo-palatine peut être dûe à une insuffisance ou une incompétence du voile [5]. Le diagnostic de l'insuffisance VP peut être effectué par l'évaluation de la parole perceptive à l'aide soit de la vidéo-fluoroscopie (VFS) ou de la naso-endoscopie (NE) [6]. Les effets primaires de l'insuffisance VP sont la fuite de débit d'air et l'hyper-nasonnement. Les effets secondaires sont les troubles de l'articulation de parole (distorsions, substitutions et omissions) [7]. Par conséquent, l'association d'une thérapeutique orthophonique à toute attitude thérapeutique principale qu'elle soit prothétique ou chirurgicale est d'une grande importance 
pour affiner au maximum ces perturbations liées au défaut vélo-palatin.

De point de vue terminologique, il faut différencier l'insuffisance VP de l'incompétence VP, la première est souvent liée à un défaut congénital ou acquis, tandis que la seconde est due généralement à une sclérose du voile en rapport avec une pathologie générale spécifique [8]. Si toutefois le traitement chirurgical de l'insuffisance VP n'est pas considéré comme une option, la gestion est réalisée au moyen d'un obturateur du pharynx ; l'incompétence VP est, en revanche, traditionnellement gérée par une prothèse de soulèvement vélo-palatine [9]. Cette prothèse obturatrice restaure les défauts du palais mou et permet une fermeture adéquate de sphincter VP. Une prothèse efficace doit pouvoir restaurer la parole, permettre une déglutition convenable, et rétablir un aspect esthétique satisfaisant. Cependant, elle doit disposer d'une rétention et d'une stabilité suffisantes [10]. Chez les patients dentés et partiellement édentés la rétention et la stabilité de la prothèse vélo-palatine sont facilement obtenues par les dents existantes [11]. Alors que chez les patients complètement édentés comme c'est le cas pour notre situation clinique l'équilibre prothétique s'avère très délicat. En effet, une maîtrise des techniques d'empreinte est d'une grande importance pour pouvoir enregistrer avec précision la surface d'appui. Les éléments anatomiques favorables à la rétention prothétique en l'occurrence les contre-dépouilles situées au niveau de la perte de substance, au niveau tubérositaire et au niveau crestal doivent être raisonnablement exploitées [12].

Le connecteur vélaire doit être mince pour le confort du patient et pour minimiser le réflexe nauséaux. Cependant, un obturateur vélo-pharyngé (OVP) connecté à l'aide de résine acrylique peut se rompre à partir de la prothèse dentaire dans la zone du connecteur vélaire, en raison du grand porte-à-faux et le poids en conjonction avec l'action dynamique vélo-pharyngée de la musculature, le risque de déglutition est possible [13]. Cet obturateur s'étend latéralement et en haut sur les parois latérales et postérieure du pharynx pour aider à la fermeture du vélo-pharynx et contribue à son fonctionnement normal [14]. Un espace d'air est laissé pour équilibrer la résonance orale et nasale
[15]. Le connecteur vélaire est généralement renforcé au moyen d'un fil ou d'un cadre en métal coulé, qui est incorporé dans la résine acrylique. Toutefois, l'augmentation de l'épaisseur de la combinaison de la résine acrylique et le fil ou le cadre métallique coulé peut perturber et gêner l'action de langue [16].

\section{CONCLUSION}

Les objectifs qu'on a pu atteindre à travers cette réhabilitation (Fig. 33) sont l'amélioration de l'esthétique par le respect des paramètres d'esthétique spécifique de la prothèse amovible complète, l'augmentation de la résonance phonatoire par l'obturation étanche de la communication bucco-sinusienne et du sphincter vélo-pharyngien, ce qui va permette aussi la suppression des fuites de liquide et de nourriture entre la cavité orale et nasale, la facilité de respiration par la reconstruction du plancher des fosses nasales, l'optimisation de la fonction masticatoire et surtout le soulagement psychique et l'intégration sociale du patient.

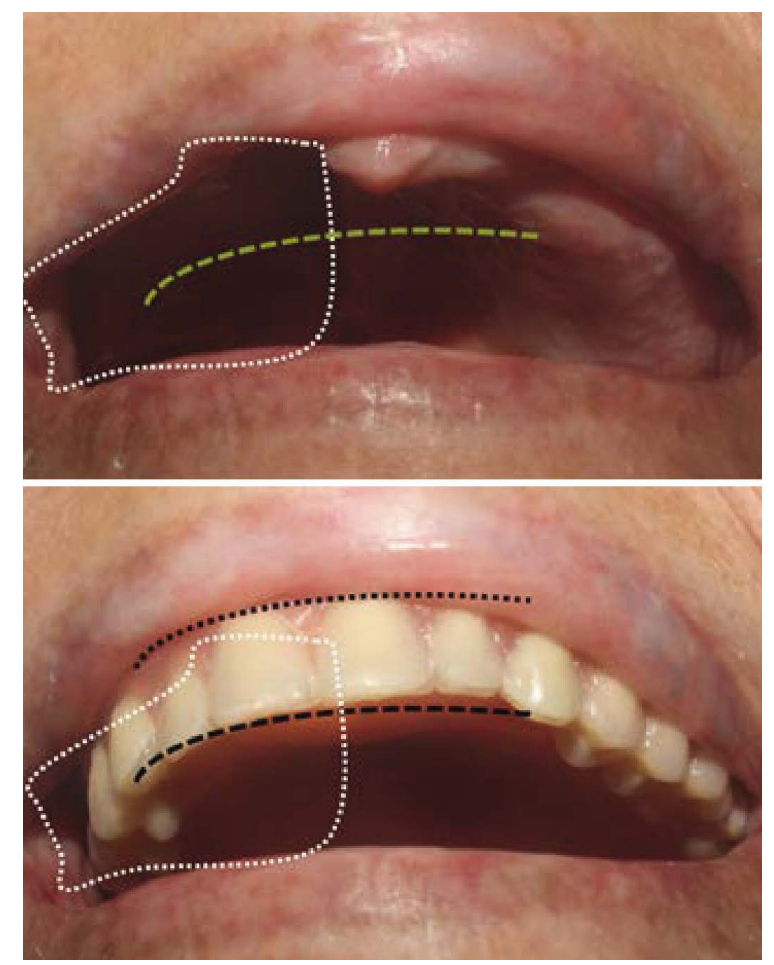

$\triangle$ Fig. 33 : Résultats esthétique et fonctionnel de la réhabilitation. 


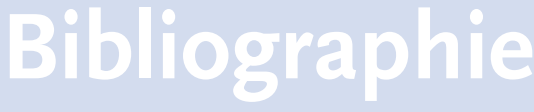

[1] Kolb F. Reconstruction en chirurgie cervico-faciale. In Brasnu D. Traité d'ORL. Flammarion édit, Paris, 2008. P. 875.

[2] Kreef A. Oral function after maxillectomy and reconstruction with an obturator. Int J Oral Maxillofac Surg $2012 ; 41$ : 1387-92.

[3] Fen-Huey L. Prosthodontic rehabilitation for edentulous patients with palatal defect : report of two cases. J Formos Med Assoc 2011 ; 110(2): 120-124.

[4] Chau-Hsiang W. Reconstruction of the soft palate in oral cancer to repair an operative defect with speech aid prosthesis : A case report. Kaohsiung J Med Sci 2007 ; 23 : 536-40.

[5] Nafees U. À new simple evaluation method of the monosyllable /sa/ using a psychoacoustic system in maxillectomy patients. J Prosth Res $2011 ; 55: 7-11$.
[6] Yen-Liang C. Preliminary study of acoustic analysis for evaluating speechaid oral prostheses : characteristic dips in octave spectrum for comparison of nasality. J Formosan Med Assoc 2014 ; 23: 1-9.

[7] Ali Ibrahim A. Palatal liftng prosthesis and velo-pharyngeal insuffisency : preliminary report. Acta Medica Academica 2013 ; 42(1): 55-60.

[8] Jurel Sunit K. Prosthetic rehabilitation of velo-pharyngeal insufficiency : a case of pharyngeal obturator with palatal lift prosthesis. JPFA $2012 ; 26$ : 53-8.

[9] Suleyman H. Prosthetic rehabilitation of velo-pharyngeal insufficiency : pharyngeal obturator prostheses with different retention mechanisms. Eur J Dent 2010 ; 4(1): 81-7.

[10] Mathew T. Intra oral scanning of hard and soft tissues for partial removable dental prosthesis fabrication. J Prosthet Dent 2014 ; 112 : 444-8.

[11] Ceyhun C. Prosthetic rehabilitation of maxillary dento-alveolar defects with fixed dental prostheses : Two clinical reports. J Prosthet Dent 2014 ; 11(2): 418- 22.

[12] Seiko H. Complete denture with a removable palatal lift prosthesis : A case report and clinical evaluation. Quint Intern $2002 ; 33$ : 675-678.

[13] Mehmet C. Prosthetic rehabilitation of a patient with a unilateral cleft palate : A clinical report J Prosthet Dent $2014 ; 111$ : 269- 272.

[14] Varun A. Conventional prosthodontic management of partial edentulism with a resilient attachment-retained overdenture in a patient with a cleft lip and palate : A clinical report. J Prosthet Dent $2014 ; 112$ : 117-121.

[15] Satoshi W. Use of palatal augmentation prostheses to maintain speech and swallowing function in patients with amyotrophic lateral sclerosis. J Oral Maxillofac Surg $2012 ; 24$ : 119-123.

[16] Murat Y. Prosthetic treatment of congenital hard and soft palate defects. Cleft Palate-Craniofacial J 2012 ; 49 (5): 618-621. 